\title{
TRADE-OFFS IN ANALOG CIRCUIT DESIGN
}

The Designer's Companion 


\title{
Trade-Offs in Analog Circuit Design
}

\section{The Designer's Companion}

\author{
Edited by \\ Chris Toumazou \\ Imperial College, UK \\ George Moschytz \\ ETH-Zentrum, Switzerland \\ and \\ Barrie Gilbert \\ Analog Devices, USA \\ Editing Assistance \\ Ganesh Kathiresan
}

KLUWER ACADEMIC PUBLISHERS

NEW YORK, BOSTON, DORDRECHT, LONDON, MOSCOW 
eBook ISBN: $\quad$ 0-306-47673-8

Print ISBN: $\quad 1-4020-7037-3$

(C2002 Kluwer Academic Publishers

New York, Boston, Dordrecht, London, Moscow

Print (C2002 Kluwer Academic Publishers

Dordrecht

All rights reserved

No part of this eBook may be reproduced or transmitted in any form or by any means, electronic, mechanical, recording, or otherwise, without written consent from the Publisher

Created in the United States of America

Visit Kluwer Online at:

http://kluweronline.com

and Kluwer's eBookstore at:

http://ebooks.kluweronline.com 


\section{Contents}

Foreword $\quad$ xxiii

List of Contributors $\quad$ xxix

\section{Design Methodology}

1

Intuitive Analog Circuit Design

Chris Toumazou

1.1 Introduction 1

1.2 The Analog Dilemma 2

$\begin{array}{ll}\text { References } & 6\end{array}$

2

Design for Manufacture $\quad 7$

Barrie Gilbert

2.1 Mass-Production of Microdevices

2.2 Unique Challenges of Analog Design 11

$\begin{array}{ll}\text { 2.2.1 Analog is Newtonian } & 13\end{array}$

$\begin{array}{ll}2.3 & \text { Designing with Manufacture in Mind } \\ & 2.3 .14\end{array}$

$\begin{array}{lll}\text { 2.3.1 Conflicts and Compromises } & 15\end{array}$

$\begin{array}{ll}\text { 2.3.2 Coping with Sensitivities: DAPs, TAPs and STMs } & 16\end{array}$

2.4 Robustness, Optimization and Trade-Offs 22

2.4.1 Choice of Architecture $\quad 25$

$\begin{array}{ll}\text { 2.4.2 Choice of Technology and Topology } & 27\end{array}$

$\begin{array}{ll}\text { 2.4.3 Remedies for Non-Robust Practices } & 32\end{array}$

2.4.4 Turning the Tables on a Non-Robust Circuit: A Case Study 34

$\begin{array}{ll}\text { Holistic optimization of the LNA } & 39\end{array}$

A further example of biasing synergy $\quad 44$

2.4.5 Robustness in Voltage References $\quad 50$

2.4.6 The Cost of Robustness $\quad 54$

2.5 Toward Design Mastery $\quad 55$

2.5.1 First, the Finale $\quad 56$

$\begin{array}{ll}\text { 2.5.2 Consider All Deliverables } & 57\end{array}$

$\begin{array}{ll}\text { 2.5.3 Design Compression } & 58\end{array}$

$\begin{array}{ll}\text { 2.5.4 Fundamentals before Finesse } & 61\end{array}$

2.5.5 Re-Utilization of Proven Cells $\quad 62$

2.5.6 Try to Break Your Circuits 63

2.5.7 Use Corner Modeling Judiciously 64

$\begin{array}{lll}\text { 2.5.8 Use Large-Signal Time-Domain Methods } & 68\end{array}$

2.5.9 Use Back-Annotation of Parasitics $\quad 68$

2.5.10 Make Your Intentions Clear $\quad 69$

$\begin{array}{ll}2.5 .11 & \text { Dubious Value of Check Lists }\end{array}$

2.5.12 Use the "Ten Things That Will Fail” Test 72

$\begin{array}{lll}2.6 & \text { Conclusion } & 73\end{array}$ 


\section{General Performance}

3

Trade-Offs in CMOS VLSI Circuits

Andrey V. Mezhiba and Eby G. Friedman

$\begin{array}{lll}3.1 & \text { Introduction } & 75\end{array}$

$\begin{array}{ll}3.2 & \text { Design Criteria } \\ & 3.2 .18\end{array}$

$\begin{array}{lll}3.2 .1 & \text { Area } & 78\end{array}$

$\begin{array}{lll}3.2 .2 & \text { Speed } & 79\end{array}$

$\begin{array}{lll}3.2 .3 & \text { Power } & 79\end{array}$

$\begin{array}{ll}3.2 .4 & \text { Design Productivity }\end{array}$

$\begin{array}{lll}3.2 .5 & \text { Testability } & 81\end{array}$

$\begin{array}{lll}3.2 .6 & \text { Reliability } & 81\end{array}$

$\begin{array}{llr}3.2 .7 & \text { Noise Tolerance } & 82\end{array}$

$\begin{array}{lll}3.2 .8 & \text { Packaging } & 83\end{array}$

$\begin{array}{lll}\text { 3.2.9 } & \text { General Considerations } & 83\end{array}$

Power dissipation in CMOS VLSI circuits $\quad 84$

$\begin{array}{lr}\text { Technology scaling } & 85\end{array}$

VLSI design methodologies $\quad 86$

3.3 Structural Level $\quad 86$

$\begin{array}{llr}\text { 3.3.1 Parallel Architecture } & 87\end{array}$

$\begin{array}{lr}\text { 3.3.2 Pipelining } & 88\end{array}$

$\begin{array}{llr}3.4 & \text { Circuit Level } & 89\end{array}$

$\begin{array}{lll}3.4 .1 & \text { Static versus Dynamic } & 90\end{array}$

$\begin{array}{lll}3.4 .2 & \text { Transistor Sizing } & 91\end{array}$

$\begin{array}{lll}3.4 .3 & \text { Tapered Buffers } & 95\end{array}$

$\begin{array}{llr}3.5 & \text { Physical Level } & 99\end{array}$

$\begin{array}{llr}3.6 & \text { Process Level } & 102\end{array}$

$\begin{array}{lll}\text { 3.6.1 Scaling } & 103\end{array}$

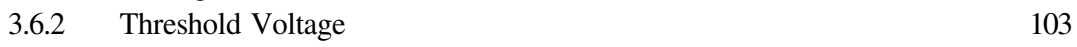

$\begin{array}{lll}3.6 .3 & \text { Power Supply } & 103\end{array}$

$\begin{array}{ll}\text { 3.6.4 Improved Interconnect and Dielectric Materials } & 104\end{array}$

$\begin{array}{lll}3.7 & \text { Future Trends } & 104\end{array}$

$\begin{array}{lr}\text { Glossary } & 107\end{array}$

$\begin{array}{ll}\text { References } & 108\end{array}$

4

$\begin{array}{ll}\text { Floating-gate Circuits and Systems } & 115\end{array}$

Tor Sverre Lande

$\begin{array}{lll}4.1 & \text { Introduction } & 115\end{array}$

$\begin{array}{ll}4.2 \text { Device Physics } & 115\end{array}$

$\begin{array}{ll}4.2 .1 & 116\end{array}$

$\begin{array}{lr}4.2 .2 & 116\end{array}$

$\begin{array}{ll}4.2 .3 & 117\end{array}$

$\begin{array}{lll}4.3 & \text { Programming } & 117\end{array}$

$\begin{array}{lll}\text { 4.3.1 UV-conductance } & 118\end{array}$

$\begin{array}{ll}\text { 4.3.2 Fowler-Nordheim Tunneling } & 118\end{array}$

$\begin{array}{lll}\text { 4.3.3 Hot Carrier Injection } & 119\end{array}$ 
$\begin{array}{lll}4.4 & \text { Circuit Elements } & 119\end{array}$

$\begin{array}{ll}\text { 4.4.1 Programming Circuits } & 120\end{array}$

$\begin{array}{ll}\text { Inter-poly tunneling } & 120\end{array}$

Example: Floating-gate on-chip knobs $\quad 121$

Inter-poly UV-programming $\quad 121$

MOS-transistor UV-conductance $\quad 122$

Example: MOS transistor threshold tuning 123

Combined programming techniques 124

Example: Single transistor synapse $\quad 126$

High-voltage drivers 127

$\begin{array}{lll}\text { 4.5 FGMOS Circuits and Systems } & 128\end{array}$

$\begin{array}{ll}\text { 4.5.1 Autozero Floating-Gate Amplifier } & 128\end{array}$

4.5.2 Low-power/Low-voltage Rail-to-Rail Circuits Using FGUVMOS 130

$\begin{array}{ll}\text { Digital FGUVMOS circuits } & 130\end{array}$

Low-voltage rail-to-rail FGUVMOS amplifier $\quad 130$

4.5.3 Adaptive Retina 132

$\begin{array}{lll}\text { 4.5.4 Other Circuits } & 134\end{array}$

$\begin{array}{lll}4.6 & \text { Retention } & 134\end{array}$

$\begin{array}{ll}4.7 & \text { Concluding Remarks } \\ & 134\end{array}$

$\begin{array}{ll}\text { References } & 135\end{array}$

5

Bandgap Reference Design 139

Arie van Staveren, Michiel H. L. Kouwenhoven, Wouter A. Serdijn and Chris

J. M. Verhoeven

$\begin{array}{lll}5.1 & \text { Introduction } & 139\end{array}$

$\begin{array}{ll}\text { 5.2 The Basic Function } & 140\end{array}$

$\begin{array}{lll}5.3 & \text { Temperature Behavior of } V_{\mathrm{BE}} & 140\end{array}$

$\begin{array}{lll}5.4 & \text { General Temperature Compensation } & 141\end{array}$

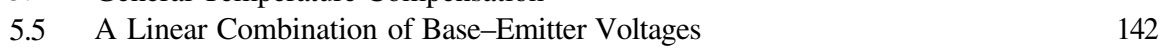

$\begin{array}{lll}\text { 5.5.1 First-Order Compensation } & 143\end{array}$

$\begin{array}{lll}\text { 5.5.2 Second-Order Compensation } & 144\end{array}$

$\begin{array}{lll}5.6 & \text { The Key Parameters } & 146\end{array}$

$\begin{array}{lll}5.7 & \text { Temperature-Dependent Resistors } & 147\end{array}$

$\begin{array}{lll}5.8 & \text { Noise } & 148\end{array}$

5.8.1 Noise of the Idealized Bandgap Reference $\quad 150$

5.8.2 Noise of a First-Order Compensated Reference 151

5.8.3 Noise of a Second-Order Compensated Reference 152

5.8.4 Power-Supply Rejection 153

$\begin{array}{lll}5.9 & \text { Simplified Structures } & 155\end{array}$

5.9.1 First-Order Compensated Reference 155

5.9.2 Second-Order Compensated Reference 156

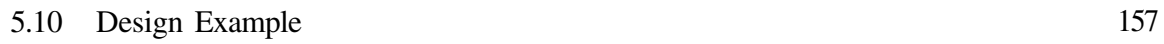

5.10.1 First-Order Compensated Bandgap Reference 157

5.10.2 Second-Order Compensated Bandgap Reference 159

$\begin{array}{lll}5.11 \text { Conclusions } & 163\end{array}$

$\begin{array}{ll}\text { References } & 164\end{array}$ 
6

Generalized Feedback Circuit Analysis

Scott K. Burgess and John Choma, Jr.

6.1 Introduction 169

6.2 Fundamental Properties of Feedback Loops 171

6.2.1 Open Loop System Architecture and Parameters 171

6.2.2 Closed Loop System Parameters 173

$\begin{array}{ll}\text { 6.2.3 Phase Margin } & 176\end{array}$

$\begin{array}{ll}\text { 6.2.4 Settling Time } & 179\end{array}$

6.3 Circuit Partitioning 182

6.3.1 Generalized Circuit Transfer Function 183

6.3.2 Generalized Driving Point I/O Impedances 189

6.3.3 Special Controlling/Controlled Port Cases 191

Controlling feedback variable is the circuit output variable 192

Global feedback 193

Controlling feedback variable is the branch variable of the controlled port 195

References

7

Analog Amplifiers Architectures: Gain Bandwidth Trade-Offs 207

Alison J. Burdett and Chris Toumazou

$\begin{array}{lll}7.1 & \text { Introduction } & 207\end{array}$

7.2 Early Concepts in Amplifier Theory 208

7.2.1 The Ideal Amplifier 208

$\begin{array}{lll}\text { 7.2.2 Reciprocity and Adjoint Networks } & 209\end{array}$

7.2.3 The Ideal Amplifier Set 210

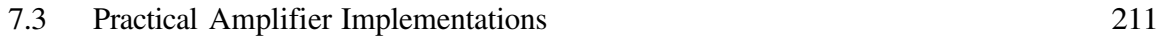

$\begin{array}{lll}\text { 7.3.1 Voltage Op-Amps } & 211\end{array}$

7.3.2 Breaking the Gain-Bandwidth Conflict 213

$\begin{array}{ll}\text { Current-feedback op-amps } & 213\end{array}$

$\begin{array}{ll}\text { Follower-based amplifiers } & 214\end{array}$

$\begin{array}{ll}\text { Current-conveyor amplifiers } & 214\end{array}$

7.3.3 Producing a Controlled Output Current 215
Clod

$\begin{array}{ll}7.4 \text { Closed-Loop Amplifier Performance } & 217\end{array}$

$\begin{array}{lll}\text { 7.4.1 Ideal Amplifiers } & 217\end{array}$

$\begin{array}{lll}\text { 7.4.2 Real Amplifiers } & 218\end{array}$

$\begin{array}{lll}7.5 & \text { Source and Load Isolation } & 222\end{array}$

$\begin{array}{lll}7.6 & \text { Conclusions } & 224\end{array}$

$\begin{array}{ll}\text { References } & 225\end{array}$

8

Noise, Gain and Bandwidth in Analog Design 227

Robert G. Meyer

$\begin{array}{lll}\text { 8.1 Gain-Bandwidth Concepts } & 227\end{array}$

8.1.1 Gain-Bandwidth Shrinkage 230

8.1.2 Gain-Bandwidth Trade-Offs Using Inductors 232

$\begin{array}{lll}8.2 & \text { Device Noise Representation } & 234\end{array}$

8.2.1 Effect of Inductors on Noise Performance 238

$\begin{array}{lll}8.3 & \text { Trade-Offs in Noise and Gain-Bandwidth } & 240\end{array}$ 
8.3.1 Methods of Trading Gain for Bandwidth and the Associated Noise Performance Implications [8]

8.3.2 The Use of Single-Stage Feedback for the Noise-Gain-Bandwidth Trade-Off

8.3.3 Use of Multi-Stage Feedback to Trade-Off Gain, Bandwidth and Noise Performance

9

Frequency Compensation

Arie van Staveren, Michiel H. L. Kouwenhoven, Wouter A. Serdijn and Chris J. M. Verhoeven

9.1 Introduction

$\begin{array}{ll}9.2 \text { Design Objective } & 258\end{array}$

$\begin{array}{ll}\text { 9.3 The Asymptotic-Gain Model } & 260\end{array}$

9.4 The Maximum Attainable Bandwidth 260

9.4.1 The LP Product 261

9.4.2 The Group of Dominant Poles 263

$\begin{array}{lll}9.5 & \text { Pole Placement } & 265\end{array}$

$\begin{array}{lll}\text { 9.5.1 Resistive Broadbanding } & 268\end{array}$

$\begin{array}{ll}\text { 9.5.2 Pole-Zero Cancelation } & 270\end{array}$

$\begin{array}{lll}9.5 .3 & \text { Pole Splitting } & 272\end{array}$

$\begin{array}{lll}\text { 9.5.4 Phantom Zeros } & 275\end{array}$

9.5.5 Order of Preference 277

$\begin{array}{ll}\text { 9.6 Adding Second-Order Effects } & 277\end{array}$

$\begin{array}{lll}9.7 & \text { Example Design } & 278\end{array}$

$\begin{array}{lll}9.8 \text { Conclusion } & 281\end{array}$

$\begin{array}{ll}\text { References } & 281\end{array}$

10

Frequency-Dynamic Range-Power 283

Eric A. Vittoz and Yannis P. Tsividis

$\begin{array}{lll}10.1 & \text { Introduction } & 283\end{array}$

10.2 Fundamental Limits of Trade-Off 284

10.2.1 Absolute Lower Boundary 284

$\begin{array}{lll}10.2 .2 & \text { Filters } & 286\end{array}$

10.2.3 Oscillators 288

10.2.4 Voltage-to-Current and Current-to-Voltage Conversion 292

10.2.5 Current Amplifiers 295

10.2.6 Voltage Amplifiers $\quad 297$

$\begin{array}{ll}10.3 \text { Process-Dependent Limitations } & 299\end{array}$

10.3.1 Parasitic Capacitors $\quad 299$

10.3.2 Additional Sources of Noise 300

10.3.3 Mismatch of Components 301

$\begin{array}{ll}\text { 10.3.4 Charge Injection } & 301\end{array}$

10.3.5 Non-Optimum Supply Voltage 302

$\begin{array}{ll}10.4 \text { Companding and Dynamic Biasing } & 303\end{array}$

$\begin{array}{lll}\text { 10.4.1 Syllabic Companding } & 303\end{array}$

10.4.2 Dynamic Biasing 306 
10.4.3 Performance in the Presence of blockers 308

10.4.4 Instantaneous Companding $\quad 309$

$\begin{array}{lll}10.5 & \text { Conclusion } & 310\end{array}$

$\begin{array}{ll}\text { References } & 311\end{array}$

\section{Filters}

11

Trade-Offs in Sensitivity, Component Spread and Component Tolerance in Active Filter Design

George Moschytz

11.1 Introduction

11.2 Basics of Sensitivity Theory 316

11.3 The Component Sensitivity of Active Filters 319

$\begin{array}{lll}11.4 & \text { Filter Selectivity, Pole } Q \text { and Sensitivity } & 325\end{array}$

$\begin{array}{ll}11.5 & \text { Maximizing the Selectivity of RC Networks } \\ 11.6 & 328\end{array}$

11.6 Some Design Examples 332

$\begin{array}{lll}11.7 & \text { Sensitivity and Noise } & 337\end{array}$

$\begin{array}{lll}11.8 & \text { Summary and Conclusions } & 339\end{array}$

References $\quad 339$

12

Continuous-Time Filters 341

Robert Fox

12.1 Introduction 341

12.2 Filter-Design Trade-Offs: Selectivity, Filter Order, Pole $Q$ and
Transient Response

12.3 Circuit Trade-Offs 342

12.3.1 Linearity vs Tuneability $\quad 342$

12.3.2 Passive Components $\quad 342$

12.3.3 Tuneable Resistance Using MOSFETs: The MOSFET-C Approach 343

12.4 The Transconductance-C (Gm-C) Approach 344

12.4.1 Triode-Region Transconductors 345

12.4.2 Saturation-Region Transconductors $\quad 346$

12.4.3 MOSFETs Used for Degeneration $\quad 346$

$\begin{array}{lll}\text { 12.4.4 BJT-Based Transconductors } & 347\end{array}$

$\begin{array}{lll}\text { 12.4.5 Offset Differential Pairs } & 347\end{array}$

12.5 Dynamic Range 347

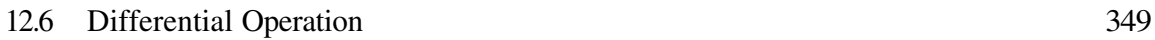

$\begin{array}{lll}12.7 & \text { Log-Domain Filtering } & 349\end{array}$

12.8 Transconductor Frequency-Response Trade-Offs 350

12.9 Tuning Trade-Offs 351

Notuning $\quad 352$

Off-chip tuning $\quad 352$

One-time post-fabrication tuning $\quad 352$

Automatictuning $\quad 352$

12.10 Simulation Issues 353

References $\quad 353$ 
13

Insights in Log-Domain Filtering

Emmanuel M. Drakakis and Alison J. Burdett

13.1 General 355

13.2 Synthesis and Design of Log-Domain Filters 360

13.3 Impact of BJT Non-Idealities upon Log-Domain Transfer Functions:

The Lowpass Biquad Example 374

13.4 Floating Capacitor-Based Realization of Finite Transmission Zeros in

Log-Domain: The Impact upon Linearity 380

13.5 Effect of Modulation Index upon Internal Log-Domain Current Bandwidth 383

13.6 Distortion Properties of Log-Domain Circuits: The Lossy Integrator Case 390

13.7 Noise Properties of Log-Domain Circuits: The Lossy Integrator Case 393

$\begin{array}{lll}13.8 & \text { Summary } & 401\end{array}$

$\begin{array}{ll}\text { References } & 401\end{array}$

\section{Switched Circuits}

14

Trade-offs in the Design of CMOS Comparators

A. Rodríguez-Vázquez, M. Delgado-Restituto, R. Domínguez-Castro, F. Medeiro and J.M. de la Rosa

14.1 Introduction

14.2 Overview of Basic CMOS Voltage Comparator Architectures

1421 Single-Step Voltage Comparators

14.2 Multistep Comparators 412

14.2.3 Regenerative Positive-Feedback Comparators 417

14.2.4 Pre-Amplified Regenerative Comparators 421

14.3 Architectural Speed vs Resolution Trade-Offs 423

14.3.1 Single-Step Comparators $\quad 423$

14.3.2 Multistep Comparators $\quad 425$

14.3.3 Regenerative Comparators 426

14.4 On the impact of the offset 429

$\begin{array}{ll}14.5 & \text { Offset-Compensated Comparators } \\ \end{array}$

14.5.1 Offset-Compensation Through Dynamic Biasing 433

14.5.2 Offset Compensation in Multistep Comparators 435

14.5.3 Residual Offset and Gain Degradation in Self-Biased Comparators 436

14.5.4 Transient Behavior and Dynamic Resolution in Self-Biased

Comparators

$\begin{array}{lr}14.6 \text { Appendix. Simplified MOST Model } & 438 \\ \text { References } & 439\end{array}$

15

Switched-Capacitor Circuits $\quad 443$

Andrea Baschirotto

$\begin{array}{lll}15.1 & \text { Introduction } & 443\end{array}$

15.2 Trade-Off due to Scaled CMOS Technology 445

15.2.1 Reduction of the MOS Output Impedance $\left(r_{0}\right) \quad 446$

15.2.2 Increase of the Flicker Noise 447

15.2.3 Increase of the MOS Leakage Current 447

15.2.4 Reduction of the Supply Voltage 448 
15.3 Trade-Off in High-Frequency SC Circuits 451

15.3.1 Trade-Off Between an IIR and a FIR Frequency Response 452

15.3.2 Trade-Off in SC Parallel Solutions 453

15.3.3 Trade-Off in the Frequency Choice 454

$\begin{array}{lll}15.4 & \text { Conclusions } & 456\end{array}$

Acknowledgments $\quad 456$

References $\quad 457$

16

Compatibility of SC Technique with Digital VLSI Technology 461

Kritsapon Leelavattananon and Chris Toumazou

$\begin{array}{lll}16.1 & \text { Introduction } & 461\end{array}$

16.2 Monolithic MOS Capacitors Available in Digital VLSI Processes 461

16.2.1 Polysilicon-over-Polysilicon (or Double-Poly) Structure 462

16.2.2 Polysilicon-over-Diffusion Structure 462

16.2.3 Metal-over-Metal Structure 463

16.2.4 Metal-over-Polysilicon Structure 464

16.2.5 MOSFET Gate Structure 464

16.3 Operational Amplifiers in Standard VLSI Processes 466

16.3.1 Operational Amplifier Topologies 466

Single-stage (telescopic) amplifier 466

Folded cascode amplifier $\quad 466$

Gain-boosting amplifier $\quad 467$

Two-stage amplifier $\quad 468$

16.3.2 Frequency Compensation $\quad 469$

Miller compensation $\quad 469$

Miller compensation incorporating source follower $\quad 470$

$\begin{array}{ll}\text { Cascode Miller Compensation } & 471\end{array}$

16.3.3 Common-Mode Feedback 472

$\begin{array}{lll}16.4 & \text { Charge-Domain Processing } & 474\end{array}$

16.5 Linearity Enhanced Composite Capacitor Branches 477

16.5.1 Series Compensation Capacitor Branch 480

16.5.2 Parallel Compensation Capacitor Branch 482

16.5.3 Balanced Compensation Capacitor Branch 483

16.6 Practical Considerations 485

16.6.1 Bias Voltage Mismatch $\quad 485$

16.6.2 Capacitor Mismatch 485

16.6.3 Parasitic Capacitances 486

$\begin{array}{lll}16.7 & \text { Summary } & 487\end{array}$

$\begin{array}{ll}\text { References } & 488\end{array}$

17

Switched-Capacitors or Switched-Currents - Which Will Succeed? 491

John Hughes and Apisak Worapishet

$\begin{array}{lll}17.1 & \text { Introduction } & 491\end{array}$

$\begin{array}{lll}17.2 & \text { Test Vehicles and Performance Criteria } & 492\end{array}$

$\begin{array}{lll}17.3 \text { Clock Frequency } & 494\end{array}$

17.3.1 Switched-Capacitor Settling $\quad 495$

17.3.2 Switched-Currents Class A Settling 497

17.3.3 Switched-Currents Class AB Settling 498 
17.4 Power Consumption

17.4.1 Switched-Capacitors and Switched-Currents Class A Power Consumption

17.4.2 Switched-Currents Class AB Power Consumption 499

17.5 Signal-to-Noise Ratio

17.5.1 Switched-Capacitors Noise

17.5.2 Switched-Currents Class A Noise

503

17.5.3 Switched-Current Class AB Noise

506

17.5.4 Comparison of Signal-to-Noise Ratios

17.6 Figure-of-Merit

$\begin{array}{lll}\text { 17.6.1 Switched-Capacitors } & 509\end{array}$

$\begin{array}{lll}\text { 17.6.2 Switched-Currents Class A } & 510\end{array}$

17.6.3 Switched-Currents Class AB $\quad 510$

$\begin{array}{lll}17.7 & \text { Comparison of Figures-of-Merit } & 510\end{array}$

$\begin{array}{lll}17.8 \text { Conclusions } & 514\end{array}$

$\begin{array}{ll}\text { References } & 514\end{array}$

\section{Oscillators}

18

Design of Integrated LC VCOS

Donhee Ham

18.1 Introduction

18.2 Graphical Nonlinear Programming 518

18.3 LC VCO Design Constraints and an Objective Function 519

18.3.1 Design Constraints $\quad 522$

18.3.2 Phase Noise as an Objective Function $\quad 522$

18.3.3 Phase Noise Approximation $\quad 523$

18.3.4 Independent Design Variables $\quad 525$

18.4 LC VCO Optimization via GNP $\quad 526$

18.4.1 Example of Design Constraints $\quad 527$

18.4.2 GNP with a Fixed Inductor $\quad 527$

18.4.3 GNP with a Fixed Inductance Value $\quad 530$

18.4.4 Inductance and Current Selection 533

18.4.5 Summary of the Optimization Process 535

18.4.6 Remarks on Final Adjustment and Robust Design 536

18.5 Discussion on LC VCO Optimization 537

$\begin{array}{lll}18.6 \text { Simulation } & 540\end{array}$

18.7 Experimental Results $\quad 541$

$\begin{array}{lll}18.8 \text { Conclusion } & 545\end{array}$

Acknowledgments $\quad 546$

References $\quad 546$

19

Trade-Offs in Oscillator Phase Noise $\quad 551$

Ali Hajimiri

$\begin{array}{lll}19.1 & \text { Motivation } & 551\end{array}$

19.2 Measures of Frequency Instability $\quad 551$

19.2.1 Phase Noise 554

19.2.2 Timing Jitter 556 
$\begin{array}{lll}19.3 & \text { Phase Noise Modeling } & 557\end{array}$

19.3.1 Up-Conversion of $1 / f$ Noise $\quad 562$

$\begin{array}{ll}\text { 19.3.2 Time-Varying Noise Sources } & 563\end{array}$

19.4 Phase Noise Trade-Offs in LC Oscillators $\quad 565$

19.4.1 Tank Voltage Amplitude $\quad 565$

19.4.2 Noise Sources $\quad 570$

Stationary noise approximation $\quad 570$

Cyclostationary noise sources $\quad 572$

19.4.3 Design Implications $\quad 573$

19.5 Phase Noise Trade-Offs for Ring Oscillators 574

19.5.1 The Impulse Sensitivity Function for Ring Oscillators 574

19.5.2 Expressions for Phase Noise in Ring Oscillators 579

19.5.3 Substrate and Supply Noise $\quad 582$

19.5.4 Design Trade-Offs in Ring Oscillators 584

References $\quad 585$

\section{Data Converters}

20

Systematic Design of High-Performance Data Converters

Georges Gielen, Jan Vandenbussche, Geert Van der Plas, Walter Daems, Anne Van den Bosch, Michiel Steyaert and Willy Sansen

$\begin{array}{lll}20.1 & \text { Introduction } & 591\end{array}$

20.2 Systematic Design Flow for D/A Converters 592

20.3 Current-Steering D/A Converter Architecture 594

20.4 Generic Behavioral Modeling for the Top-Down Phase 597

$\begin{array}{ll}20.5 \text { Sizing Synthesis of the D/A Converter } & 599\end{array}$

$\begin{array}{ll}\text { 20.5.1 Architectural-Level Synthesis } & 600\end{array}$

$\begin{array}{ll}\text { Static performance } & 600\end{array}$

$\begin{array}{ll}\text { Dynamic performance } & 601\end{array}$

20.5.2 Circuit-Level Synthesis $\quad 602$

$\begin{array}{ll}\text { Static performance } & 602\end{array}$

$\begin{array}{ll}\text { Dynamic performance } & 603\end{array}$

20.5.3 Full Decoder Synthesis $\quad 603$

20.5.4 Clock Driver Synthesis 603

$\begin{array}{lll}20.6 & \text { Layout Synthesis of the D/A Converter } & 603\end{array}$

20.6.1 Floorplanning $\quad 604$

20.6.2 Circuit and Module Layout Generation 604

$\begin{array}{ll}\text { Current-source array layout generation } & 604\end{array}$

$\begin{array}{ll}\text { Swatch array layout generation } & 605\end{array}$

Full decoder standard cell place and route $\quad 605$

20.6.3 Converter Layout Assembly 606

20.7 Extracted Behavioral Model for Bottom-Up Verification 606

$\begin{array}{lll}20.8 & \text { Experimental Results } & 607\end{array}$

$\begin{array}{lll}20.9 \text { Conclusions } & 610\end{array}$

$\begin{array}{ll}\text { Acknowledgments } & 610\end{array}$

$\begin{array}{ll}\text { References } & 610\end{array}$ 
21

Analog Power Modeling for Data Converters and Filters

Georges Gielen and Erik Lauwers

$\begin{array}{lll}21.1 & \text { Introduction } & 613\end{array}$

21.2 Approaches for Analog Power Estimators 614

21.3 A Power Estimation Model for High-Speed Nyquist-Rate ADCs 616

21.3.1 The Power Estimator Derivation 616

21.3.2 Results of the Power Estimator 619

21.4 A Power Estimation Model for Analog Continuous-Time Filters 620

$\begin{array}{lll}21.4 .1 & \text { The ACTIF Approach } & 620\end{array}$

21.4.2 Description of the Filter Synthesis Part 621

21.4.3 OTA Behavioral Modeling and Optimization for Minimal Power Consumption 624

Modeling of the transconductances $\quad 624$

The distortion model $\quad 625$

$\begin{array}{ll}\text { Optimization } & 626\end{array}$

21.4.4 Experimental Results $\quad 627$

$\begin{array}{lll}21.5 & \text { Conclusions } & 627\end{array}$

$\begin{array}{ll}\text { Acknowledgment } & 628\end{array}$

$\begin{array}{ll}\text { References } & 628\end{array}$

22

Speed vs. dynamic range Trade-Off in Oversampling Data Converters 631

Richard Schreier, Jesper Steensgaard and Gabor C. Temes

22.1 Introduction 631

$\begin{array}{lll}22.2 & \text { Oversampling Data Converters } & 632\end{array}$

22.2.1 Quantization Error $\quad 632$

22.2.2 Feedback Quantizers 633

22.2.3 Oversampling D/A Converters $\quad 636$

22.2.4 Oversampling A/D Converters 639

22.2.5 Multibit Quantization $\quad 640$

$\begin{array}{lll}22.3 & \text { Mismatch Shaping } & 644\end{array}$

22.3.1 Element Rotation $\quad 644$

22.3.2 Generalized Mismatch-Shaping 645

22.3.3 Other Mismatch-Shaping Architectures 649

22.3.4 Performance Comparison 650

$\begin{array}{lll}22.4 & \text { Reconstructing a Sampled Signal } & 653\end{array}$

22.4.1 The Interpolation Process $\quad 654$

An interpolation system example $\quad 654$

22.4.2 Fundamental Architectures for Practical Implementations 656

Single-bit delta-sigma modulation $\quad 657$

Multibit delta-sigma modulation 657

High-resolution oversampled D/A converters 658

22.4.3 High-Resolution Mismatch-Shaping D/A Converters 659

A fresh look on mismatch shaping $\quad 659$

$\begin{array}{ll}\text { Practical implementations } & 660\end{array}$

$\begin{array}{ll}\text { References } & 662\end{array}$ 


\section{Transceivers}

23

Power-Conscious Design of Wireless Circuits and Systems 665

Asad A. Abidi

23.1 Introduction

23.2 Lowering Power across the Hierarchy 667

23.3 Power Conscious RF and Baseband Circuits 668

23.3.1 Dynamic Range and Power Consumption 668

23.3.2 Lowering Power in Tuned Circuits 670

23.3.3 Importance of Passives Quality in Resonant Circuits 671

$\begin{array}{ll}\text { 23.3.4 Low Noise Amplifiers } & 673\end{array}$

$\begin{array}{lll}23.3 .5 & \text { Oscillators } & 678\end{array}$

$\begin{array}{lll}\text { 23.3.6 Mixers } & 681\end{array}$

$\begin{array}{lll}\text { 23.3.7 } & \text { Frequency Dividers } & 685\end{array}$

23.3.8 Baseband Circuits $\quad 686$

23.3.9 On-Chip Inductors $\quad 689$

23.3.10 Examples of Low Power Radio Implementations 691

23.3.11 Conclusions: Circuits $\quad 692$

$\begin{array}{ll}\text { References } & 692\end{array}$

24

$\begin{array}{ll}\text { Photoreceiver Design } & 697\end{array}$

Mark Forbes

$\begin{array}{lll}24.1 & \text { Introduction } & 697\end{array}$

24.2 Review of Receiver Structure 698

$\begin{array}{lll}24.3 & \text { Front-End Small-Signal Performance } & 700\end{array}$

$\begin{array}{lll}24.3 .1 & \text { Small-Signal Analysis } & 700\end{array}$

24.3.2 Speed/Sensitivity Trade-Off $\quad 702$

24.3.3 Calculations, for example, parameters 706

$\begin{array}{lll}24.4 & \text { Noise Limits } & 707\end{array}$

$\begin{array}{lll}24.5 & \text { Post-Amplifier Performance } & 709\end{array}$

24.6 Front-End and Post-Amplifier Combined Trade-Off 712

$\begin{array}{lll}24.7 & \text { Mismatch } & 714\end{array}$

$\begin{array}{lll}24.8 \text { Conclusions } & 718\end{array}$

$\begin{array}{lr}\text { Acknowledgments } & 718\end{array}$

$\begin{array}{ll}\text { References } & 719\end{array}$

25

Analog Front-End Design Considerations for DSL 723

Nianxiong Nick Tan

$\begin{array}{lll}25.1 & \text { Introduction } & 723\end{array}$

$\begin{array}{lll}25.2 & \text { System Considerations } & 725\end{array}$

25.2.1 Digital vs Analog Process 725

25.2.2 Active vs Passive Filters 726

$\begin{array}{ll}25.3 & \text { Data Converter Requirements for DSL } \\ \end{array}$

25.3.1 Optimum Data Converters for ADSL 732

$\begin{array}{ll}\text { Optimum ADCs for ADSL } & 732\end{array}$

Optimum ADC for ADSL-CO $\quad 734$

Optimum ADC for ADSL-CP $\quad 735$ 
Optimum DACs $\quad 735$

$\begin{array}{ll}\text { Optimum DAC for ADSL-CO } & 737\end{array}$

Optimum DAC for ADSL-CP 737

$\begin{array}{lll}\text { 25.3.2 Function of Filtering } & 738\end{array}$

$\begin{array}{lll}25.4 & \text { Circuit Considerations } & 740\end{array}$

25.4.1 Oversampling vs Nyquist Data Converters $\quad 740$

25.4.2 SI vs SC 743

25.4.3 Sampled-Data vs Continuous-Time Filters 743

$\begin{array}{lll}25.4 .4 & \text { Gm-C vs RC filters } & 744\end{array}$

$\begin{array}{lll}25.5 & \text { Conclusions } & 744\end{array}$

$\begin{array}{ll}\text { Acknowledgments } & 745\end{array}$

$\begin{array}{ll}\text { References } & 745\end{array}$

26

Low Noise Design $\quad 747$

Michiel H. L. Kouwenhoven, Arie van Staveren, WouterA. Serdijn and

Chris J. M. Verhoeven

$\begin{array}{lll}26.1 & \text { Introduction } & 747\end{array}$

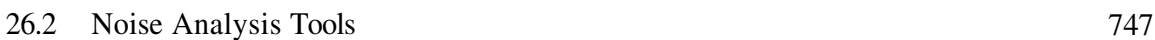

$\begin{array}{lll}26.2 .1 & \text { Equivalent Noise Source } & 748\end{array}$

26.2.2 Transform-I: Voltage Source Shift $\quad 749$

26.2.3 Transform-II: Current Source Shift 749

26.2.4 Transform-III: Norton-Thévenin Transform 749

26.2.5 Transform-IV: Shift through Twoports $\quad 750$

26.3 Low-Noise Amplifier Design 751

26.3.1 Design of the Feedback Network 752

Noise production by the feedback network 753

Magnification of nullor noise 754

Distortion increment and bandwidth reduction $\quad 755$

26.3.2 Design of the Active Part for Low Noise 756

26.3.3 Noise Optimizations $\quad 757$

Noise matching to the source $\quad 757$

Optimization of the bias current $\quad 759$

$\begin{array}{ll}\text { Connecting stages in series/parallel } & 760\end{array}$

$\begin{array}{ll}\text { Summary of optimizations } & 761\end{array}$

26.4 Low Noise Harmonic Resonator Oscillator Design 762

26.4.1 General Structure of a Resonator Oscillator 762

26.4.2 Noise Contribution of the Resonator 763

26.4.3 Design of the Undamping Circuit for Low Noise 764

Principle implementation of the undamping circuit $\quad 765$

Amplitude control $\quad 765$

Noise performance $\quad 766$

Driving the oscillator load 766

26.4.4 Noise Matching of the Resonator and Undamping Circuit: Tapping 767

26.4.5 Power Matching $\quad 769$

26.4.6 Coupled Resonator Oscillators $\quad 770$

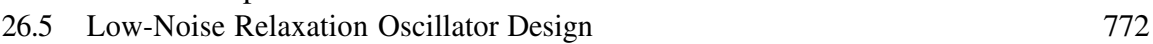

26.5.1 Phase Noise in Relaxation Oscillators 773

Simple phase noise model $\quad 773$ 
Influence of the memory on the oscillator phase noise $\quad 774$

Influence of comparators on the oscillator phase noise 776

26.5.2 Improvement of the Noise Behavior by Alternative Topologies 777

$\begin{array}{ll}\text { Relaxation oscillators with memory bypass } & 778\end{array}$

$\begin{array}{ll}\text { Coupled relaxation oscillators } & 780\end{array}$

$\begin{array}{ll}\text { References } & 784\end{array}$

27

Trade-Offs in CMOS Mixer Design $\quad 787$

$\begin{array}{ll}\text { Ganesh Kathiresan and Chris Toumazou } & \\ 27.1 \quad \text { Introduction } & 787\end{array}$

27.1.1 The RF Receiver Re-Visited 788

$\begin{array}{lll}27.2 & \text { Some Mixer Basics } & 789\end{array}$

$\begin{array}{lll}\text { 27.2.1 Mixers vs Multipliers } & 789\end{array}$

27.2.2 Mixers: Nonlinear or Linear-Time-Variant? 791

$\begin{array}{lll}27.3 & \text { Mixer Figures of Merit } & 792\end{array}$

27.3.1 Conversion Gain and Bandwidth 793

27.3.2 $1 \mathrm{~dB}$ Compression Point $\quad 794$

27.3.3 Third-Order Intercept Point 796

$\begin{array}{lll}\text { 27.3.4 Noise Figure } & 797\end{array}$

27.3.5 Port-to-Port Isolation $\quad 799$

27.3.6 Common Mode Rejection, Power Supply, etc 799

$\begin{array}{lll}27.4 & \text { Mixer Architectures and Trade-Offs } & 800\end{array}$

27.4.1 Single Balanced Differential Pair Mixer $\quad 800$

27.4.2 Double-Balanced Mixer and Its Conversion Gain 803

27.4.3 Supply Voltage $\quad 805$

Active loads $\quad 805$

$\begin{array}{lr}\text { Inductive current source } & 805\end{array}$

$\begin{array}{lr}\text { Two stack source coupled mixer } & 806\end{array}$

$\begin{array}{lr}\text { Bulk driven topologies } & 807\end{array}$

$\begin{array}{lll}27.4 .4 & \text { Linearity } & 809\end{array}$

Source degeneration $\quad 809$

Switched MOSFET degeneration $\quad 811$

$\begin{array}{lll}\text { 27.4.5 LO Feedthrough } & 812\end{array}$

$\begin{array}{lll}27.4 .6 & \text { Mixer Noise } & 813\end{array}$

Noise due to the load $\quad 814$

$\begin{array}{ll}\text { Noise due to the input transconductor } & 814\end{array}$

Noise due to the switches $\quad 815$

$\begin{array}{lll}27.5 & \text { Conclusion } & 817\end{array}$

$\begin{array}{ll}\text { References } & 817\end{array}$

28

A High-performance Dynamic-logic Phase-Frequency Detector 821

Shenggao Li and Mohammed Ismail

$\begin{array}{lll}28.1 & \text { Introduction } & 821\end{array}$

28.2 Phase Detectors Review $\quad 822$

$\begin{array}{lll}28.2 .1 & \text { Multiplier } & 822\end{array}$

28.2.2 Exclusive-OR Gate $\quad 823$ 
28.2.3 JK-Flipflop $\quad 825$

28.2.4 Tri-State Phase Detector $\quad 825$

28.3 Design Issues in Phase-Frequency Detectors 827

$\begin{array}{lll}\text { 28.3.1 Dead-Zone } & 827\end{array}$

$\begin{array}{ll}\text { 28.3.2 Blind-Zone } & 829\end{array}$

28.4 Dynamic Logic Phase-Frequency Detectors 831

28.5 A Novel Dynamic-Logic Phase-Frequency Detector 835

28.5.1 Circuit Operation 836

28.5.2 Performance Evaluation 837

$\begin{array}{ll}28.6 \text { Conclusion } & 842\end{array}$

$\begin{array}{lr}\text { References } & 842\end{array}$

29

Trade-Offs in Power Amplifiers 843

Chung Kei Thomas Chan, Steve Hung-Lung Tu and Chris Toumazou
29.1 Introduction

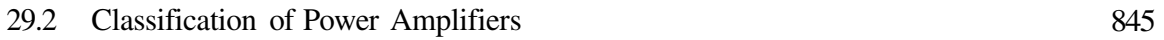

29.2.1 Current-Source Power Amplifiers $\quad 845$

29.2.2 Switch-Mode Power Amplifiers 848

$\begin{array}{ll}\text { Class D power amplifier } & 848\end{array}$

Class E power amplifier $\quad 849$

Class F power amplifier $\quad 850$

29.2.3 Bandwidth Efficiency, Power Efficiency and Linearity 852

29.3 Effect of Loaded $Q$-Factor on Class E Power Amplifiers 853

29.3.1 Circuit Analysis 853

29.3.2 Power Efficiency $\quad 857$

29.3.3 Circuit Simulation and Discussion $\quad 858$

29.4 Class E Power Amplifiers with Nonlinear Shunt Capacitance 861

29.4.1 Numerical Computation of Optimum Component Values 863

Basic equations $\quad 863$

Optimum operation (Alinikula's method [16]) 865

$\begin{array}{ll}\text { Fourier analysis } & 869\end{array}$

$\begin{array}{lr}\text { Normalized power capability } & 869\end{array}$

29.4.2 Generalized Numerical Method $\quad 870$

$\begin{array}{ll}\text { Design example } & 872\end{array}$

$\begin{array}{ll}\text { Small linear shunt capacitor } & 872\end{array}$

$\begin{array}{lll}29.5 & \text { Conclusion } & 878\end{array}$

$\begin{array}{lr}\text { References } & 880\end{array}$

\section{Neural Processing}

30

Trade-Offs in Standard and Universal CNN Cells $\quad 883$

Martin Hänggi, Radu Dogaru and Leon O. Chua

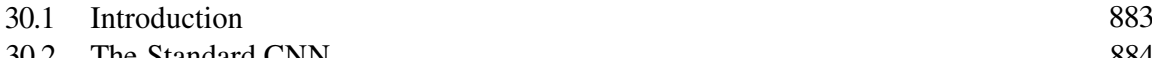

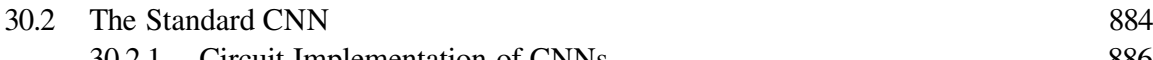

30.2.1 Circuit Implementation of CNNs 886

30.3 Standard CNN Cells: Robustness vs Processing Speed 887

30.3.1 Reliability of a Standard CNN 887 
$\begin{array}{ll}\text { Introduction } & 887\end{array}$

Absolute and relative robustness $\quad 888$

The Robustness of a CNN template set $\quad 888$

$\begin{array}{lr}\text { Template scaling } & 890\end{array}$

Template design $\quad 890$

30.3.2 The Settling Time of a Standard CNN 892

Introduction $\quad 892$

The exact approach for uncoupled CNNS 893

30.3.3 Analysis of Propagation-Type Templates 893

$\begin{array}{ll}\text { Introduction } & 893\end{array}$

Examples of propagation-type templates $\quad 894$

30.3.4 Robust CNN Algorithms for High-Connectivity Tasks 897

$\begin{array}{ll}\text { Template classes } & 898\end{array}$

One-step vs algorithmic processing 900

30.3.5 Concluding Remarks 901

30.4 Universal CNN Cells and their Trade-Offs 902

30.4.1 Preliminaries $\quad 902$

30.4.2 Pyramidal CNN cells 904

Architecture 904

Trade-offs 905

30.4.3 Canonical Piecewise-linear CNN cells 906

Characterization and architecture 906

Trade-offs $\quad 907$

Example 908

30.4.4 The Multi-Nested Universal CNN Cell 909

Architecture and characterization $\quad 909$

$\begin{array}{ll}\text { Trade-offs } & 910\end{array}$

30.4.5 An RTD-Based Multi-Nested Universal CNN Cell Circuit 914

$\begin{array}{ll}\text { 30.4.6 Concluding Remarks } & 917\end{array}$

$\begin{array}{lr}\text { References } & 918\end{array}$

\section{Analog CAD}

\section{1}

Top-Down Design Methodology For Analog Circuits Using Matlab and Simulink 923 Naveen Chandra and Gordon W. Roberts

$\begin{array}{lll}31.1 & \text { Introduction } & 923\end{array}$

31.2 Design Methodology Motivation 925

31.2.1 Optimization Procedure 926

31.3 Switched Capacitor Delta-Sigma Design Procedure 927

31.3.1 Switched Sampled Capacitor (kT/C) Noise 928

31.3.2 OTA Parameters $\quad 929$

31.4 Modeling of $\Delta \Sigma$ Modulators in Simulink $\quad 929$

31.4.1 Sampled Capacitor (kT/C) Noise $\quad 930$

31.4.2 OTA Noise 931

31.4.3 Switched Capacitor Integrator Non-Idealities $\quad 932$

$\begin{array}{lll}31.5 & \text { Optimization Setup } & 938\end{array}$

$\begin{array}{lll}\text { 31.5.1 Implementation in Matlab } & 941\end{array}$

$\begin{array}{lll}\text { 31.5.2 Initial Conditions } & 943\end{array}$

$\begin{array}{ll}\text { 31.5.3 Additional Factors } & 945\end{array}$ 
31.6 Summary of Simulation Results 945

31.7 A Fully Coded $\Delta \Sigma$ Modulator Design Example 946

31.8 Conclusion $\quad 950$

References $\quad 951$

32

Techniques and Applications of Symbolic Analysis for Analog Integrated Circuits

Georges Gielen

32.1 Introduction 953

$\begin{array}{ll}32.2 & \text { What is Symbolic Analysis? } \\ & 953\end{array}$

32.2.1 Definition of Symbolic Analysis 953

32.2.2 Basic Methodology of Symbolic Analysis 956

32.3 Applications of Symbolic Analysis 958

32.3.1 Insight into Circuit Behavior 958

32.3.2 Analytic Model Generation for Automated Analog Circuit Sizing 960

32.3.3 Interactive Circuit Exploration 961

32.3.4 Repetitive Formula Evaluation $\quad 961$

32.3.5 Analog Fault Diagnosis $\quad 962$

32.3.6 Behavioral Model Generation 963

32.3.7 Formal Verification $\quad 964$

32.3.8 Summary of Applications 965

32.4 Present Capabilities and Limitations of Symbolic Analysis 965

32.4.1 Symbolic Approximation 966

32.4.2 Improving Computational Efficiency 968

32.4.3 Simplification During Generation 969

32.4.4 Simplification Before Generation $\quad 971$

32.4.5 Hierarchical Decomposition 971

32.4.6 Symbolic Pole-Zero Analysis 974

32.4.7 Symbolic Distortion Analysis $\quad 974$

32.4.8 Open Research Topics 976

32.5 Comparison of Symbolic Simulators 976

$\begin{array}{lll}32.6 & \text { Conclusions } & 977\end{array}$

$\begin{array}{lr}\text { Acknowledgments } & 979\end{array}$

$\begin{array}{lr}\text { References } & 979\end{array}$

33

Topics in IC Layout for Manufacture $\quad 985$

Barrie Gilbert

33.1 Layout: The Crucial Next Step 985

33.1.1 An Architectural Analogy 988

33.1.2 IC Layout: A Matter of "Drafting"? 989

33.1.3 A Shared Undertaking 992

33.1.4 What Inputs should the Layouteer Expect? 993

$\begin{array}{lll}33.2 & \text { Interconnects } & 996\end{array}$

$\begin{array}{lll}33.2 .1 & \text { Metal Limitations } & 998\end{array}$

33.2.2 Other Metalization Trade-Offs 1000

$\begin{array}{ll}33.3 \text { Substrates and the Myth of "Ground" } & 1006\end{array}$

$\begin{array}{lll}\text { 33.3.1 Device-Level Substrate Nodes } & 1009\end{array}$

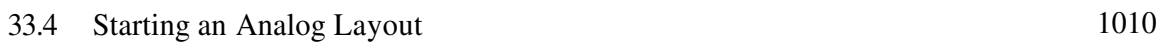


33.5 Device Matching

1012

33.5.1 The "Biggest-of-All" Layout Trade-Off

1015

33.5.2 Matching Rules for Specific Components

1016

33.5.3 Capacitor Matching

1018

33.5.4 Circuit/Layout Synergy

1020

33.6 Layout of Silicon-on-Insulator Processes

1024

33.6.1 Consequences of High Thermal Resistance

1028

33.7 Reflections on Superintegrated Layout

1029

Index

1033 


\section{Foreword}

With so many excellent texts about analog integrated circuit design now available, the need for yet another compilation of contributions may be questioned. Nevertheless, this book fills a notable void, in addressing a topic that, while a common aspect of a product designer's life, is only occasionally addressed in engineering texts. It is about TradeOffs: What they are; the circumstances in which they arise; why they are needed; how they are managed, and the many ingenious ways in which their conflicting demands can be resolved. We call it a Designer's Companion, since it is more in the nature of a reference work, to dip into when and where some new perspectives on the topic are needed, rather than a text to be read in isolation and absorbed as a whole. However, it is an aspect of a trade-off that it is peculiar to each situation and there are no recipes for their instant resolution. That being true, their treatment here is frequently by example, suggestive rather than definitive. The personal insights, intuitions and inventiveness of the designer remain vital to the pursuit of a well-balanced solution, but which is even then only one of many, so its selection requires a relative-value judgment.

Understanding how to cope with trade-offs is an indispensable and inextricable part of all engineering. In electronics, and particularly in analog design, the dilemmas arise in the choice of basic cell topology, its biasing, the specific element values and in making performance compromises. For example, wireless communication systems are becoming increasingly sophisticated: they must operate at ever higher carrier frequencies, while using increasingly complex modulation modes, and posing extremely stringent performance demands. Meeting these requirements is only made more difficult as the dimensions of transistors and passive elements in modern IC processes continue to shrink, and as time-to-market and cost pressures mount. Similar trends are found throughout the field of electronics: in power management, fiber-optics, clock generation for CPUs, high-precision instrumentation for signal generation and metrology, and in analytical equipment of numerous kinds in science, industry, medicine and more recently in forensics and security.

Simply stated, the need for a trade-off is generated by the dilemma of being faced with a multiplicity of paths forward in the design process, each providing a different set of benefits or posing different risks, and which can only be resolved by giving up certain benefits in exchange for others of comparable value. The trade-off invariably generates a constellation of considerations which are specific to each situation, within a particular design context and set of circumstances that will often have never occurred before, and whose resolution will have little general applicability.

It is these latter features that make writing about trade-offs so difficult: they are not easy to anticipate in a systematic treatment, and they don't teach lessons of universal applicability. Furthermore, a trade-off calls for creativity: it requires us to provide what isn't there, in the data. Trade-offs cannot be made by tossing a coin; they are rarely of an either-or character to begin with. The longer one mulls over the unique particulars, the more likely it is that a panoply of solutions will present themselves, to be added to 
one's bulging list of options. At some point, of course, ingenuity has to be curbed, and a decision has to be made.

Edward de Bono has noted that "In the end, all [human] decisions are emotional". In resolving a trade-off, our intervention as laterally thinking, resourceful individuals is not required if the facts unequivocally speak for themselves, that is, if the resolution of a transient dilemma can be achieved algorithmically. It involves selecting one from several similarly attractive choices. We invariably try to apply all sorts of wisdom and logic to our choice of which car or house to buy; but when logic fails to force the answer, as it so often does, we fall back on emotion. The essential role of emotion as an intrinsic part of rational intelligence and an ally to creative thought has recently been illuminated by a few pioneering psychologists. Intriguingly, in the index to Antonio Damasio's 1994 book Descartes' Error, one finds the entry "Decision making: see Emotion". Coping with trade-offs also requires the inquisitive anticipation of the circumstances in which they may arise, and a good deal of practice in playing out What If? scenarios. Joel Arthur Barker ${ }^{1}$ makes this observation, in which we may want to substitute "the next IC development" in place of "the new worlds coming":

Some anticipation can be scientific, but the most important aspect of anticipation is artistic. And, just like the artist, practice and persistence will dramatically improve your abilities. Your improved ability will, in turn, increase your ability in dealing with the new worlds coming.

[Emphases added]

Although often referred to as "an art" in casual conversation, circuit design is more correctly viewed as a craft. The central emphasis in formal treatments of integrated circuit design is generally on acquiring a thorough knowledge of the underlying electronic principles, and of semiconductor processes and devices, aided by a fluency in mathematics, familiarity with the particular domain of specialization under consideration, and a basic ability for applying various pre-packaged concepts, techniques and algorithms. But this hides the importance of developing the knack of making all the right judgments in practicing this craft, and the value of cultivating a personal flair in coping with the realities beyond the covers of the textbook.

Contrarily, from the layman's perspective, design is perceived as a linear intellectual process, which proceeds something like this: One is faced with a set of objectives, and then calls on experience to assemble all the pieces in a methodical, step-by-step fashion, making fact-driven decisions along the way. As each part of the product is considered, logic prevails at every juncture, and the whole gradually takes on a shape that is as optimal as it is inevitable, to become another testament to the power of the underlying rules and theories.

As a seasoned product designer, you will know that from the outset this will be far from the reality. Inspired guesses (more charitably labeled "engineering judgments")

1 Joel Arthur Barker, Paradigms: The Business of Discovering the Future, 1994. This highly recommended work was previously published in 1992 under the title Future Edge. By that time anything with the word "Future" in its title was already becoming passé, so perhaps it enjoyed only lackluster sales. By contrast, "Paradigms" was a very marketable word in 1994. 
are scattered all along the path, from start to finish. To begin with, those Objectives, that are supposed to inform every step of the proceedings and give the development a sure sense of direction, are either insufferably detailed and give one a feeling of being imprisoned in a straightjacket, or they are so comically sketchy and perhaps mutually inconsistent, that anything approaching a focused, optimal solution is out of the question. Regrettably, as your own experience may testify, both of these extremes are all-too common, as well as every flavour in between. Each in its own way is mischievously setting the stage for the first trade-off to be needed.

In the over-constrained scenario, one designer may be inclined to take a stab at satisfying the provider of the objectives with the desired results, no less, but no more, either: a just-right solution. This could be unwise, however, since the writer of these specifications might be viewing the development in a way that is strongly influenced by a prior discrete-element solution, and could be unaware of the special advantages that can be provided by a monolithic implementation. On the other, this tactic might be the right one if the product needs to meet only this one customer's need, and development time is severely limited, and die cost must be minimized. Another designer might adopt the opposite rationale: Sure, the product will meet all those fussy requirements, but it could be capable of doing a lot more, too. By skillful design, many additional applications and features can be anticipated, and the versatility extended to embrace these, for little extra design effort or manufacturing cost. Thus, each of these two designers is making a trade-off, right at the start, about how to interpret and react to the challenge implicit in the specifications.

Similarly, when faced with scant information about what is needed of this new product, one designer's approach might be to opt for caution, and painstakingly solicit more detailed information from the provider of the objectives. This only generates another trade-off, since the provider/user may in fact be no more informed than the designer; but, perhaps to hide his ignorance, he will nonetheless generate more numbers based on estimates and prior practice, in other words, more guesses. If these are received and acted on with unmerited respect, the outcome could be a disaster. Alternatively, if they are treated with disdain, and another set of guesses is substituted, the outcome could be equally undesirable.

Meanwhile, a second designer may lean on her specialized experience with similar products, and assume that the missing information can be adequately interpolated, without the need for any further consultation. That tactic could work out well, or it could be just the beginning of a monstrous headache for both the potential user and the designer. In all these scenarios, it is painfully evident that the tools needed for resolution of this particular dilemma will be found in no text book (including this one!) and they each in their own way call for a trade-off to be made. And this before the design has even begun.

These sketches also make us aware of the arbitrariness of the trade-off. It's an idiosyncratic response to a dilemma. The more practiced the engineer, the more likely it is that the majority of the hundreds of trade-offs that eventually will have to be made, during the course of developing even a relatively straightforward analog circuit, will be based on good judgment, and a balanced consideration of all the alternatives that came 
to mind. But we cannot say that these decisions will be entirely rational, or optimal. There are no algorithms for success.

This book covers ten subject areas: Design Methodology; Technology; General Performance; Filters; Switched Circuits; Oscillators; Data Converters; Transceivers; Neural Processing; and Analog CAD. It addresses a diversity of trade-offs ranging from such well-known couplets as frequency versus dynamic range, or gain-bandwidth vs power consumption, or settling-time vs phase-noise in PLLs, to some of the more subtle trade-offs that arise in design for robustness in manufacture and in the "polygon world" of IC layout. During its several years in development, it has transcended its original scope, becoming a designer's desktop companion while also having value as a graduate textbook, inasmuch as numerous fundamental relationships leading to design conflicts are explained, in many cases with practical examples.

Its thirty-three chapters come from a variety of sources, including some of the world's most eminent analog circuits and systems designers, to provide, for the first time, a timely and comprehensive text devoted to this important aspect of analog circuit design. Those authors who are professional designers are faced every day with difficult decisions on which the success of their products depend, and not always with all the analytic horsepower that may be demanded by some of the situations. Taken in aggregate, the trade-offs that they choose eventually shape the competitive stature and reputation of the companies for whom they work. Other authors allow themselves to take a more academic view of the nature of a trade-off, and as a group are more inclined to have greater optimism about the amenability of challenging circumstances to yield to formal approaches, and even a degree automation.

The first section on Design Methodology opens with a discussion by Toumazou about the nature and value of qualitative reasoning, in contrast to the usual emphasis in engineering on the towering importance of quantitative analysis. The underlying need for intuition, playful inventiveness and emotion in the pursuit of an engineering life is picked up by Gilbert, in Chapters 2 and 33, although the more serious focus here is nonetheless on making decisions within the context of commercial product development. In all these chapters, the sheer breadth of the field allows only an introduction to the subject matter.

The next three chapters, in the Technology section, range from the "Big Picture" of VLSI, and in particular, some of the trade-offs in CMOS circuit development, as explored by Mezhiba and Friedman, to the specific and detailed topic of bandgap voltage references, as perceived by Staveren, Kouwenhoven, Serdijn and Verhoeven (Chapter 5). Perched between these two chapters is a presentation of the less-familiar floating-gate devices and circuits that have a unique, although limited, scope of applications and might also comfortably fit into the later (and short) section on Neural Processing, in Chapter 30 of which Hanggi, Dogaru and Chua discuss specialized trade-offs in integrated neural networks.

In some cases, the emphasis is on the tension between two dominant aspects of performance. This approach is particularly evident in the five chapters about General Performance issues. A very basic trade-off is that which arises between amplifier bandwidth and gain; this is discussed by Toumazou and Payne in Chapter 7, and from a different perspective by Meyer in Chapter 8. Aspects of frequency compensation 
in integrated amplifiers is explored in Chapter 9, by Staveren, Kouwenhoven, Serdijn and Verhoeven. In amplifier design, one cannot increase bandwidth without regard for noise, and this in turn is strongly influenced by the power consumption that one can afford to assign to the amplifier. Noise and bandwidth are likewise linked by device geometry. Attempts to push bandwidth may impact DC offsets or gain accuracy in certain cases, or distortion and intermodulation in others. Thus, trade-offs are usually multi-faceted, and in a very real way, nearly all the key specifications that will appear in a product data sheet will be linked to a considerable extent. Vittoz and Tsividis face up to these harsh realities in Chapter 10.

In the section on Filters, the many conflicts and compromises that surround continuous-time active-filter design are addressed by Moschytz in Chapter 11, and by Fox in Chapter 12. The particular way in which trade-offs arise in Log-Domain (Translinear) Filters is discussed by Drakakis and Burdett in Chapter 13. The next section is about Switched Circuits in general, and includes four differing perspectives. The optimization of comparators is the focus of Chapter 14, by Rodríguez-Vázquez, Delgado-Restituto, Domínguez-Castro and de la Rosa, while a general overview of switched-capacitor circuits is presented by Baschirotto in Chapter 15, followed by a review of the compatibility of such circuits with advanced digital technologies, provided by Leelavattananon. This section closes with Chapter 17, which offers some thoughts by Hughes and Worapishet about the differences and trade-offs that arise between the standard switched-capacitor circuits that are now well established and the less well-known switched-current forms that are sometimes viewed as equally useful, in certain situations.

Communications circuits are a minefield of trade-offs, and the very stringent performance required of Oscillators are examined in the Chapters 18 and 19 of this section. In the first, by Ham, some of the special problems of maintaining low phase-noise using the relatively poor on-chip components (principally low-Q inductors and lossy varactors of limited range) are put under scrutiny. A different perspective on the same subject is provided by Hajimiri. The next three chapters, in the section on Data Converters, provide insights from the foremost exponents of these extremely important gateways between the analog and digital domains. The first, which sets forth principles for the systematic design of high-performance data converters, is authored by an impressive team composed of Gielen, Vandenbussche, Van de Plas, Daems, den Bosch, Steyaert and Sansen. The following Chapter 21 is more specialized in its approach: Gielen and Lauwers discuss particular issues of power modeling for data converters and filters. Chapter 22, authored by Schreier, Steensgaard and Temes, provides a definitive account of the fundamental trade-off between speed and dynamic range in over-sampled converters.

The focus next shifts to Transceivers, in several very different arenas. In Chapter 23, Abidi shares his considerable experience in the design of wireless circuits, and the systems of which they are an integral part, where power conservation is a dominant concern. This is followed by a review by Forbes of the design trade-offs that arise in optical receivers. Finally, Chapter 25 closes this section with some considerations for analog front-ends in digital subscriber-line systems. In all these cases, the overarching challenge is the attainment of a very high dynamic range, entailing the simultaneous 
provision of low distortion, of various disparate types, with a near-fundamental noise floor. The endless search for low noise is also featured in Chapter 26, as illuminated by Kouwenhoven, Staveren, Serdijn and Verhoeven, and again, noise and intermodulation are the central challenges in mixer design, the topic of the next chapter by Kathiresan and Toumazou. Phase detectors once bore a passing resemblance to mixers, and their close cousin, the analog multiplier; but in today's phase-locked loops, there is a more pressing need to capture both phase and frequency information. Some special techniques are presented by $\mathrm{Li}$ and Ismail. The closing chapter of this section, authored by Chan, Tu and Toumazou, looks at the trade-offs that arise in the design of various sorts of power amplifiers.

The final section is concerned with CAD for analog design. Chandra and Roberts present an overview of a design methodology for analog circuits using Matlab and Simulink, while in Chapter 32, Gielen adds a concluding word about the possibilities for using symbolic analysis tools for analog circuits.

Clearly, no book on the topic of trade-offs can ever be truly representative of the entire field of analog design, nor exhaustive in its treatment of those subjects which do get included. The primary function of any engineering text is to inform, and provide accurate and authoritative guidance of both a general and specific sort. However, as earlier suggested in this Foreword, and as these chapters testify, it is unlikely that very many general recommendations can be made regarding trade-offs, and the specialized case histories have a strictly limited scope of application. But another function of any good text is to enthuse, to inspire, to illuminate the less-explored corners of the domain, and to point the way to new perspectives on each topic. It is hoped that the material assembled here serves that objective. 


\section{List of Contributors}

Asad A. Abidi

Electrical Engineering Department

University of California

Los Angeles

USA

Email: abidi@icsl.ucla.edu

\section{Andrea Baschirotto}

Department of Innovation Engineering

University of Lecce

Via per Monteroni-73100

Lecce

Italy

Email:baschirotto@ele.unipv.it

\section{Alison J. Burdett}

Department of Electrical \& Electronics Engineering Imperial College

Exhibition Road, SW7 2BT

London

UK

Email: a.burdett@ic.ac.uk

\section{Scott K. Burgess}

Department of Electrical Engineering-Electrophysics

University of Southern California

Los Angeles, California

USA

\section{Chung Kei Thomas Chan}

Circuits and Systems Group

Imperial College of Science, Technology and Medicine UK

Email: thomas.chan@ic.ac.uk 


\section{Naveen Chandra}

Microelectronics and Computer Systems Laboratory

McGill University

Montreal, Quebec

Canada

Email: naveen@macs.ece.mcgill.ca

John Choma, Jr.

Department of Electrical Engineering-Electrophysics

University of Southern California

Los Angeles, California

USA

Email: johnc@mizar.usc .edu

\section{Leon O. Chua}

Email: chuadeecs . berkeley . edu

\section{Walter Daems}

ESAT-MICAS

Katholieke Universiteit Leuven

\section{J. M. de la Rosa}

Institute of Microelectronics of Seville

CNM-CSIC

Avda. Reina Mercedes s/n

Edif. CICA, 41012-Sevilla

Spain

\section{Delgado-Restituto}

Institute of Microelectronics of Seville

CNM-CSIC

Avda. Reina Mercedes s/n

Edif. CICA, 41012-Sevilla

Spain

\section{Radu Dogaru}

\section{R. Domínguez-Castro}

Institute of Microelectronics of Seville

CNM-CSIC

Avda. Reina Mercedes s/n

Edif. CICA, 41012-Sevilla

Spain 


\section{E. M. Drakakis}

Department of Bioengineering

Imperial College

Exhibition Road, SW7 2BX

London

UK

Email: e.drakakis@ic.ac.uk

\section{Mark Forbes}

Heriot-Watt University

Edinburgh

Scotland

Email: mforbes@cadence.com

\section{Robert Fox}

University of Florida

Florida

USA

Email: fox@tec.ufl.edu

\section{Eby G. Friedman}

Department of Electrical and Computer Engineering

University of Rochester

Rochester

New York

USA

Email: friedman@ee.rochester.edu

\section{Georges Gielen}

ESAT-MICAS

Katholieke Universiteit Leuven

Email: georges.gielen@esat.kuleuven.ac.be

\section{Barrie Gilbert}

Analog Devices Inc.

1100 NW Compton Drive

Beaverton

Oregon 97006-1994

USA

Email: barrie.gilberteanalog.com 


\author{
Ali Hajimiri \\ California Institute of Technology \\ California \\ USA \\ Email: hajimiriacaltech. edu
}

\title{
Donhee Ham
}

California Institute of Technology

California

USA

Email: donheedits.caltech.edu

\section{Martin Hänggi}

Email: haenggi@computer .org

\section{John Hughes}

Email: hughesjb@prl .research.philips.com

\section{Mohammed Ismail}

Analog VLSI Lab,

The Ohio-State University

Ohio

USA

Email: ismail@ee.eng.ohio-state.edu

\section{Ganesh Kathiresan}

Circuits and Systems Group

Department of Electrical \& Electronics Engineering Imperial College of Science, Technology and Medicine London

UK

Email: g.kathiresan@ic.ac.uk

\section{Michiel H. L. Kouwenhoven}

Electronics Research Laboratory/DIMES

Delft University of Technology

The Netherlands

Email: m.h.1.kouwenhoven@its.tudelft.ne

\section{Tor Sverre Lande}

Department of Informatics

University of Oslo

Oslo

Norway

Email: bassen@ifi.uio.no 


\section{Erik Lauwers}

ESAT-MICAS

Katholieke Universiteit Leuven

\section{Kritsapon Leelavattananon}

Ericsson Microelectronics

Swindon Design Centre

Pagoda House

Westmead Drive, Westlea

Swindon SN5 7UN

UK

Email: kritsaponaswindon.ericsson.se

\section{Shenggao Li}

Analog VLSI Lab,

The Ohio-State University

Wireless PAN Operations,

Intel Corporation, San Francisco

California

USA

Email: lisg@ee.eng.ohio-state.edu

\section{F. Madiero}

\section{Andrey V. Mezhiba}

Department of Electrical and Computer Engineering University of Rochester

Rochester

New York

USA

\section{George Moschytz}

Swiss Federal Institute of Technology

Switzerland

Email: moschytz@isi.ee.ethz.ch

\section{Gordon W. Roberts}

Microelectronics and Computer Systems Laboratory

McGill University

Montreal, Quebec

Canada

Email: roberts@macs.ece.mcgill.ca 


\section{A. Rodríguez-Vázquez}

Institute of Microelectronics of Seville

CNM-CSIC

Avda. Reina Mercedes s/n

Edif. CICA, 41012-Sevilla

Spain

\section{Willy Sansen}

ESAT-MICAS

Katholieke Universiteit Leuven

\section{Richard Schreier}

\section{Wouter A. Serdijn}

Electronics Research Laboratory/DIMES

Delft University of Technology

The Netherlands

Email: w.a.serdijnaits.tudelft.nl

\section{Arie van Staveren}

Electronics Research Laboratory/DIMES

Delft University of Technology

The Netherlands

\section{Jesper Steensgaard}

Email: steensgaard@ieee.org

\section{Michiel Steyaert}

ESAT-MICAS

Katholieke Universiteit Leuven

Nianxiong Nick Tan

GlobeSpan, Inc.

Irvine, California, USA

\section{Gabor C. Temes}

Email: temes@ece.orst.edu

\section{Chris Toumazou}

Circuits \& Systems Group

Department of Electrical Engineering

Imperial College of Science, Technology \& Medicine

London

UK

Email: c.toumazou@ic.ac.uk 


\section{Yannis P. Tsividis}

Columbia University

New York

USA

Email: tsividis@ee.columbia.edu

\section{Steve Hung-Lung Tu}

Circuits and Systems Group

Imperial College of Science, Technology and Medicine

London

UK

Anne Van den Bosch

ESAT-MICAS

Katholieke Universiteit Leuven

\section{Geert Van der Plas}

ESAT-MICAS

Katholieke Universiteit Leuven

\section{Jan Vandenbussche}

ESAT-MICAS

Katholieke Universiteit Leuven

\section{Chris J. M. Verhoeven}

Electronics Research Laboratory/DIMES

Delft University of Technology

The Netherlands

\section{Eric A. Vittoz}

Swiss Centre for Electronics and Microtechnology

Switzerland

\section{Apisak Worapishet}

